\title{
Power Quality Disturbances Classification and Recognition Using S-transform Based Neural classifier
}

\author{
P.Kalyana Sundaram ${ }^{1}$, R.Neela $^{2}$ \\ ${ }^{1}$ Assistant Professor, Electrical Engineering, Annamalai University \\ ${ }^{2}$ Professor, Electrical Engineering, Annamalai University
}

\begin{abstract}
This paper presents an automated recognition and classification technique for the various power quality disturbances. This technique is based on S-transform and MLP based neural network. The distorted voltage waveforms are generated using Matlab simulation on the test system. The S-Transform method is introduced as a powerful tool for the input feature extraction from the distorted voltage waveforms. The extracted features are applied as the inputs to the MLP based neural network for classification of various classes of power quality disturbances. For each class of disturbances MLP based neural network has been trained at the rate of 100 samples. The algorithm has been tested with 900 number of test data and the outcomes are recorded. The performance of the proposed technique has been evaluated by comparing the results against S-transform based fuzzy expert system.
\end{abstract}

Keywords: Power quality, Power quality disturbances, S-transform, Neural Network, MLP Based Neural Network.

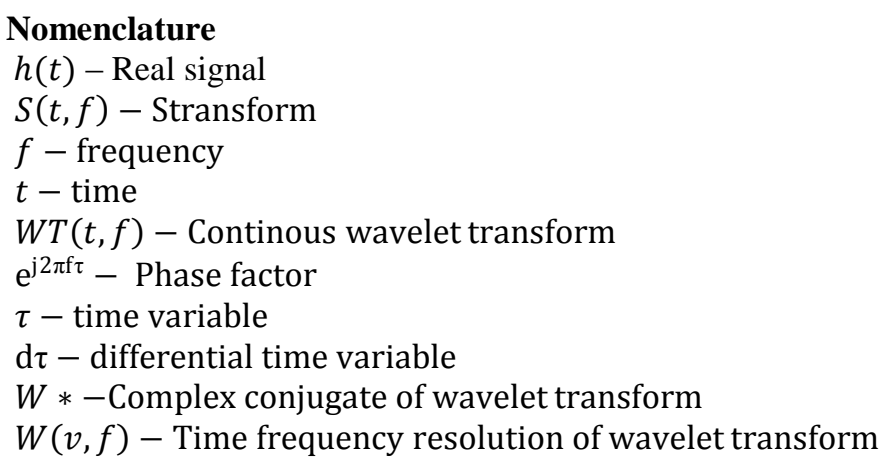

\section{Introduction}

Now a day's power quality has become a main problem in the electric power system. The reason for the poor quality of electric power is caused by the power line disturbances such as sag, swell, interruption, harmonics, sag with harmonics, swell with harmonics, flicker and notches. However in order to improve the electric power quality, the sources and occurrences of such disturbances must be detected. A large variety of power quality detection and classification tools were developed over the past few years to analyze stationary and non stationary signals in both time and frequency domain. The various types of power quality disturbances were detected and classified using wavelet transform analysis as illustrated in [1]. An adaptive neural network based power quality analyzer for the estimation of electric power quality has been applied and the disturbances were classified in [2]. Another approach of wavelets for categorize and classify the various power system disturbances has been discussed in [3]. An automated power quality monitoring and equipment modeling technique has been discussed in [4] to characterize and classify the various types of power quality events. A combination of Fourier and wavelet transform with rule based expert systems has been implemented for the characterization of power quality events in [5].

Wavelet multi resolution analysis based neural network classifier is presented in [6] for the classification of automated online power quality disturbances. The detection and classification of various types of power quality disturbances are based on S-transform have been illustrated in [7]. S-transform based neural network classifier is presented in [8] where the analysis of the non stationary signals in the power system has been presented. Artificial neural network (ANN) based real time electric power quality disturbance classification has been illustrated in [9]. A rule based system along with time frequency analysis techniques were discussed for PQ disturbance classification in [10]. Probabilistic neural network method along with Stransform based on optimal feature selection for power quality disturbances classification has been illustrated in [11]. 
A combination of Discrete wavelet transform (DWT) along with support vector machine (SVM) has been presented in [12] for the detection and analysis of power quality disturbances. As Hilbert transform shows greater immunity towards noise, it has been used for the detection and classification of different types of power quality events along with ANN in [13]. S-transform based fuzzy and PSO classifier has been presented in [14] and this identified and classified the PQ disturbance in time domain. Particle swarm optimization technique has used to identify and eliminate the harmonics current in the power system [15]. A hybrid method for the real time frequency estimation based on Taylor series and discrete Fourier algorithm has been illustrated in [16]. Classification of power quality disturbances using the combined form of Hilbert huang transform (HHT) and Relevance vector machine (RVM) has been presented in [17].A hybrid power quality monitoring technique based on Ensemble empirical mode decomposition (EEMD) and Hilbert transform (HT) using support vector machine (SVM) classifier has presented in [18].

Analysis of non linear and non stationary signal with the measurement of voltage flicker signals based on Orthogonal Hilbert Huang transform (OHHT) is illustrated in [19]. Classifications of various non stationary power quality disturbances based on EMD along with Hilbert transform and neural network has been elaborated in [20]. A S-transform and MLP neural network based power quality analyzer in which features are extracted through S-transform and disturbances are classified based on MLP neural network is presented in this paper.

\section{Proposed Method}

The proposed method has two stages namely a feature extraction stage and a classification stage. In the feature extraction stage the S-transform is used for extracting features such as peak value, variances and standard deviation. The classification stage consists of an MLP based neural network with four hidden layers.. The disturbance waveforms are generated using Matlab simulation on the test system.

\subsection{Feature Extraction Stage using S-transform}

The S-transform is a generalization of the Short-time Fourier transform (STFT) and an extension of the continuous wavelet transforms (CWT). The S-transform will perform multi-resolution analysis (MRA) on a time varying power signal, as its window width varies inversely with the frequency. The main function of the $S$ transform is a Gaussian modulation cosinusoid. The output of the S-transform is an $\mathrm{N} x \mathrm{M}$ matrix called the Smatrix whose rows pertain to the frequency and columns to time.

A spectrogram or sonogram, is a visual representation of the spectrum of frequencies. Spectrograms are usually created in one of two ways. They are approximated as a filter bank that results from a series of band pass filters, or calculated from the time signal using the short-time Fourier transform (STFT). These two methods actually form two different Time-Frequency Distributions, but are equivalent under some conditions. Stransform using spectrogram analysis provides better visual analyze of the signal.

The S-transform of a signal $h(t)$ is defined as

$$
S(t, f)=\int_{-\infty}^{\infty} h(\tau) w^{*}(\tau-t, f), e^{-j 2 \pi f \tau} d \tau
$$

Where $S(t, f)=\frac{|f|}{\alpha \sqrt{2} \pi}, e^{-t f^{2} / 2 \alpha^{2}}$

The integration of S-transform over time results in the Fourier spectrum is given as

$$
H(f)=\int_{-\infty}^{\infty} S(t, f) d t
$$

For the gaussian window $\int_{-\infty}^{\infty} S(t, f) d t=1$

The original signal can be obtained from $\mathrm{S}$-transform as

$$
h(t)=\int_{-\infty}^{\infty}\left\{\int_{-\infty}^{\infty} S(\tau, f) d \tau\right\} e^{j 2 \pi f \tau} d f
$$

The S-transform is also represent as the amplitude and phase correction of the continuous wavelet transform

$$
S(t, f)=\frac{\sqrt{f}}{2 \pi \alpha} e^{j 2 \pi f \tau}, W T(t, f)
$$

Wavelet transform is given as $W T(t, f)=\sqrt{\frac{|f|}{\alpha}}, e^{-t^{2} f^{2} / 2 \alpha^{2}}, e^{j 2 \pi f \tau}$

The $\mathrm{S}$-transform is now expressed in time frequency resolution as

$$
\begin{gathered}
S(t, f)=\int_{-\infty}^{\infty} H(v, f) w^{*}(v, f), e^{j 2 \pi v \tau} d v \\
w(v, f)=e^{-2 \pi^{2} \alpha^{2} v^{2} / t^{2}}
\end{gathered}
$$

The amplitude, phase and envelop mean value are given by

$$
\begin{aligned}
& A(t)=\frac{\sqrt{f}}{2 \pi \alpha} \\
& \tilde{A}(t)=T^{-1} \int_{0}^{T} A(t) d t
\end{aligned}
$$

The variance of the signal is the first input to fuzzy system. It is directly computed from the envelop mean value as follows 


$$
V_{a r}(t)=(S(t, f)-\tilde{A}(t))^{2}
$$

The standard deviation is the second input to fuzzy system. It is obtained from the following relationship as given below

$$
S_{t d}(t)=\sqrt{V_{a r}(t)}
$$

\subsection{Multi-Layer Perceptron (MLP) Neural Network}

A multilayer perceptron neural network is a feed-forward artificial neural network that has an input layer, output layer and one or more hidden layers. A MLP based neural network consists of multiple layers of nodes in which each layer connected to the next one fully in a directed graph. Except for the input nodes, each node is a neuron with a nonlinear activation function. MLP based neural network utilizes a supervised learning technique called back propagation for training the network. MLP based neural network architecture diagram is shown as in the figure 1.

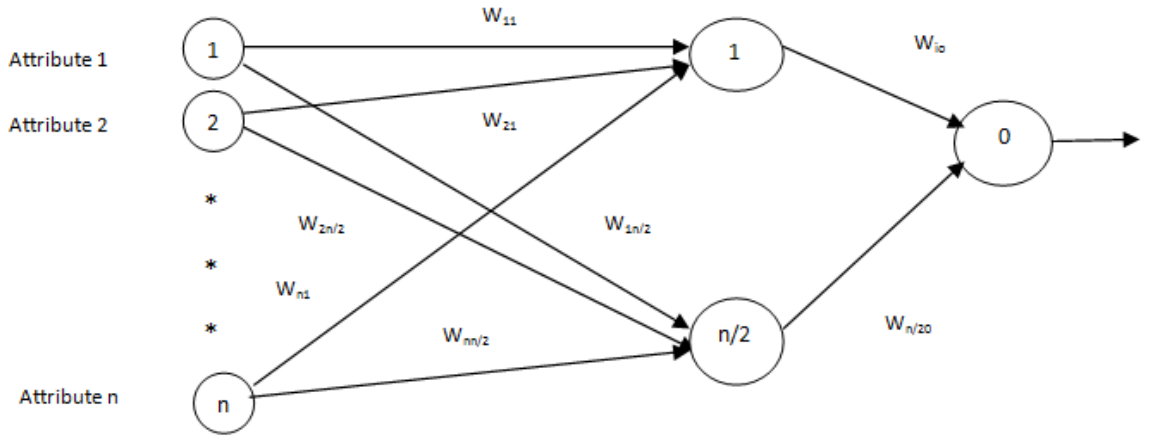

Figure 1 Architecture of MLP Neural Network

Further, MLP networks have redundant networking and are very robust, providing a mathematical flexibility. The training parameters and the structure of the MLP used in this work are shown in Table 1.

Table 1 MLP architecture and training parameters

\section{Architecture}

The number of layers

The number of neuron on the layers

The initial weights and biases

Activation functions

Training parameters

Learning rule

Learning rate

Mean-squared error
3

Input: 13, hidden: 10, output: 9

Random

Tangent sigmoid

Back-propagation

0.75

$1 \mathrm{E}-08$

\section{Back propagation learning algorithm}

BP has two phases:

$>$ Forward pass phase: Computes 'functional signal', feed forward propagation of input pattern signals through network.

$>$ Backward pass phase: Computes 'error signal', propagates the error backwards through network starting at output units (where the error is the difference between actual and desired output values).

The back-propagation network has an input layer, an output layer, and atleast one hidden layer. There is no limit on the number of hidden layers but typically there is just one or two . But in some case a minimum requirement of four layers (three hidden layers plus an output layer) are used to solve complex problems. Each layer is fully connected to the succeeding layer. Recall is the process of setting input data into a trained network and receiving the answer. Back-propagation is not used during recall, but only when the network is learning a training set.

\section{Classification Stage}

In this stage, S-transform extracts the input features such as standard deviation, peak value, variances. The extracted input features are applied to the multi-layer perception based neural network in order to classify the disturbances.MLP networks are very useful for the classification of those input signals. 


\subsection{Flowchart of the Proposed Method}

The flowchart for the Classification of Power Quality disturbances is shown in below.

It has two different blocks.

- $\quad$ Block-(a) - Features extraction such as standard deviation and variances

- Block-(b) - Detection and classification of the power quality disturbances

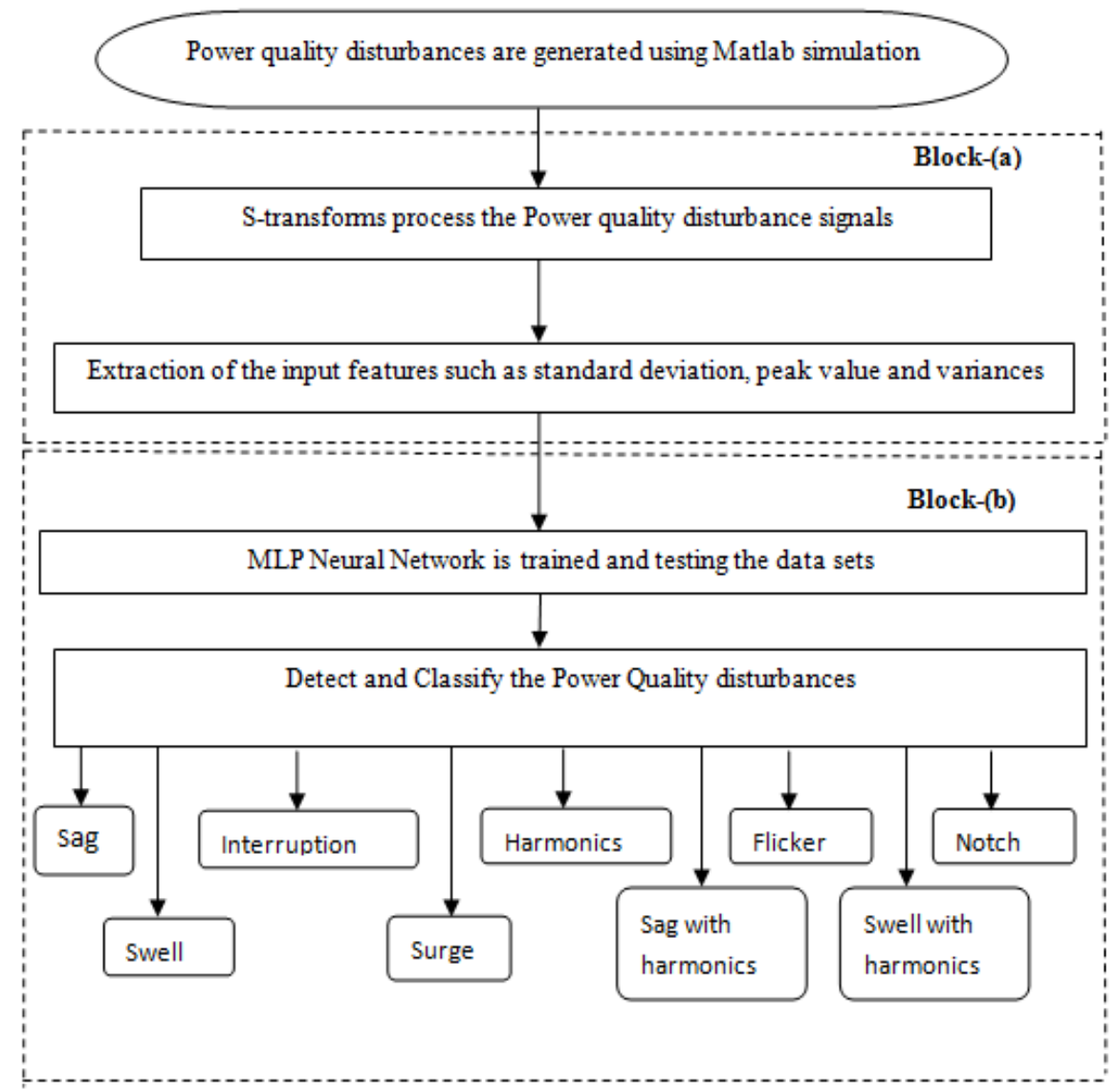

Figure 2. Flowchart for the Classification of Power Quality disturbances

\section{Simulation and Test Results}

Testing data were generated using Matlab simulink on the test system model for various classes of disturbances and the signals closer to real situation can be simulated. The nine types of different power quality disturbances, namely pure sine (normal), sag, swell, outage, harmonics, sag with harmonic, swell with harmonic, notch and flicker were considered. The single line diagram for the test system and the Matlab simulation block diagram are shown in figure 5 and figure 6.

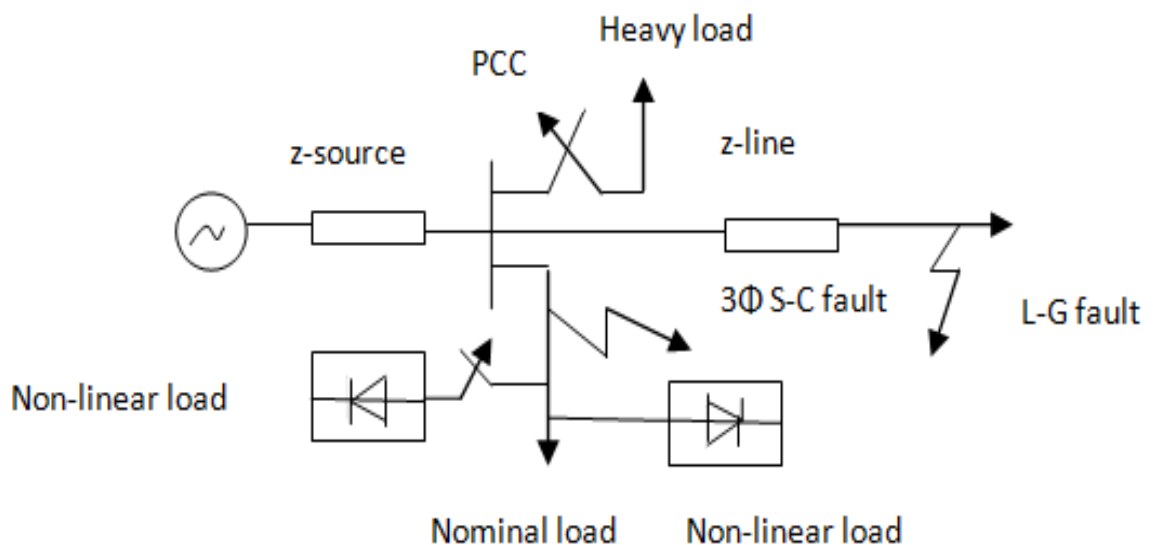

Figure 3.Single line diagram of test system model 


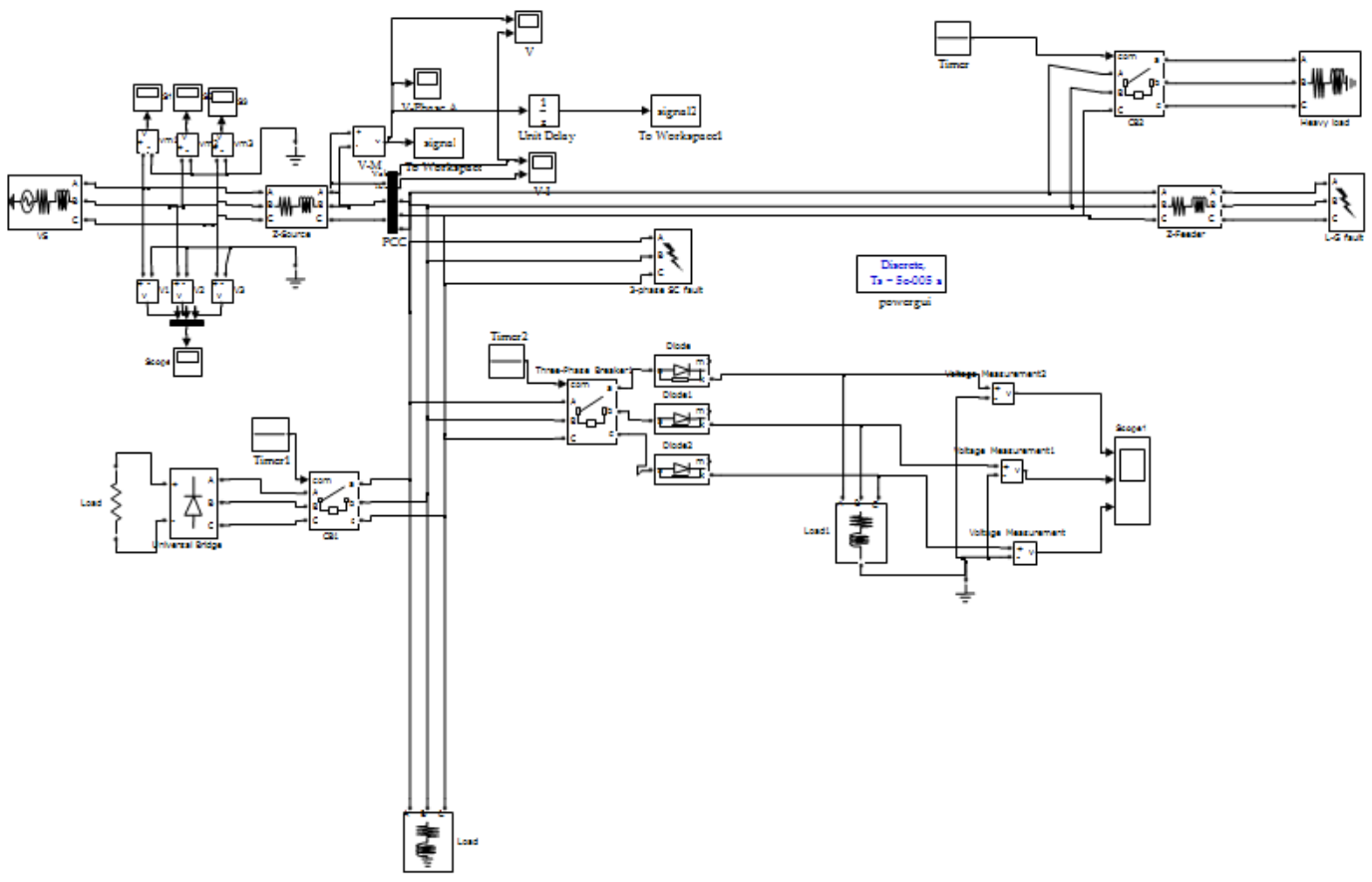

Figure 4.Matlab simulation block diagram for the test system model

Total size of the training data set is $3 * 900$, where 3 represents the number of features extracted for each type of disturbance and 900 represents the total number of samples at the rate of 100 samples for each one of the 9 disturbances. These input signals, when applied to the MLP neural network to get accurate classified disturbances. The power quality disturbance signals generated using the Matlab simulation on the test system. The following case studies are presented to highlight the suitability of the application of the proposed method.

Pure sine wave is a normal voltage signal of amplitude $1 \mathrm{~V}$ at the frequency $50 \mathrm{~Hz}$ and its waveform is as shown in the figure 5(a).The time frequency analysis of S-transform and the spectrogram representation of the pure sine wave are shown in the figures 5(b) and 5(c). The standard deviation, peak value and variances of the pure sine wave are shown in the figures 5(d) and 5(f).

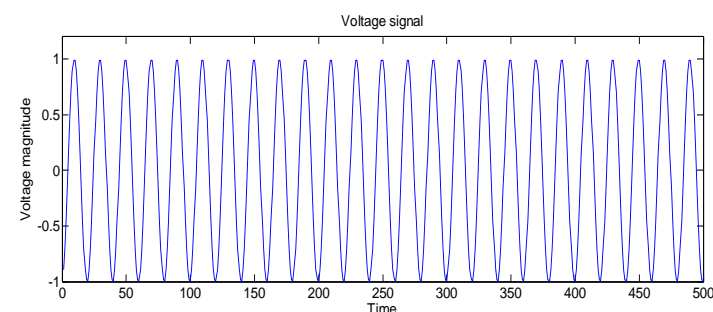

Figure 5(a)

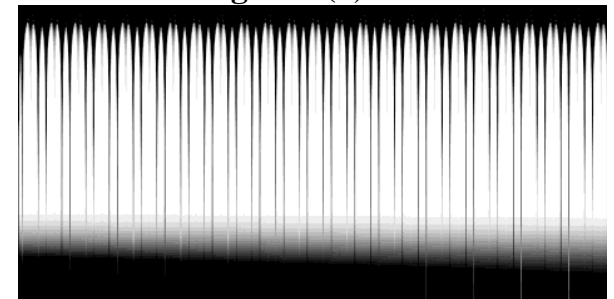

Figure 5(b)

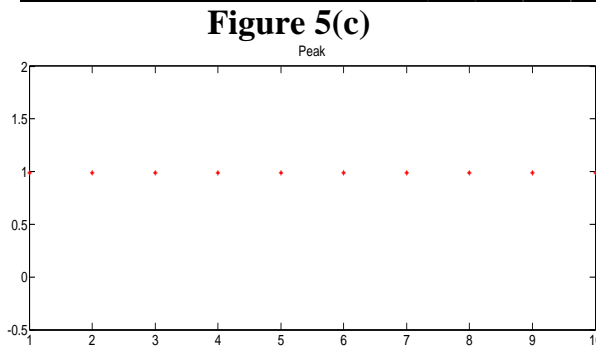

Figure 5(e)
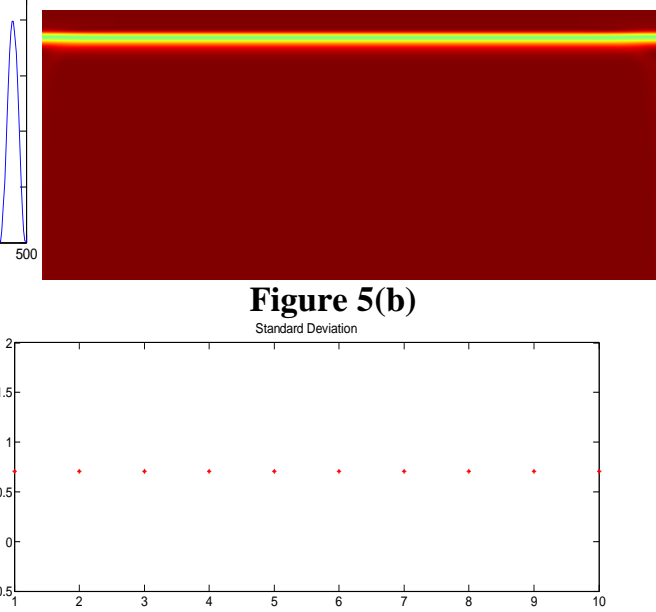

Figure 5(d)

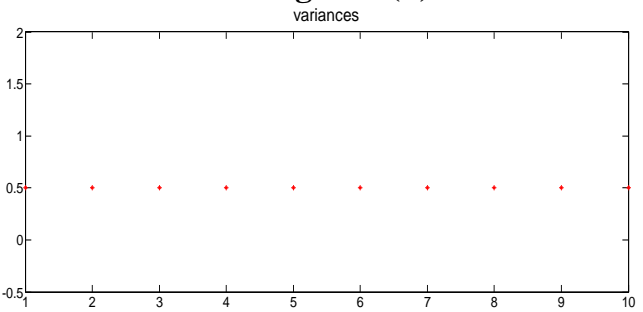

Figure 5(f) 
Voltage sag (or) voltage dips cause a decrease of $10-90 \%$ in system voltage. The duration of the sag disturbance is 0.2 to 0.4 cycles in $1 \mathrm{~min}$. It is generated by the occurrence of a single line to ground fault for 10 cycles. The voltage dip waveform is shown in the figure 6(a). The time frequency analysis of S-transform and the spectrogram representation are shown in the figures 6(b) and 6(c). The standard deviation, peak value and the variances of the voltage sag are shown in the figures $6(\mathrm{~d})$ and $6(\mathrm{f})$.

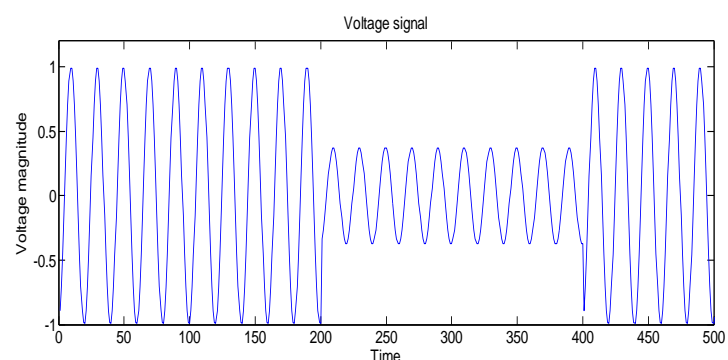

Figure 6(a)

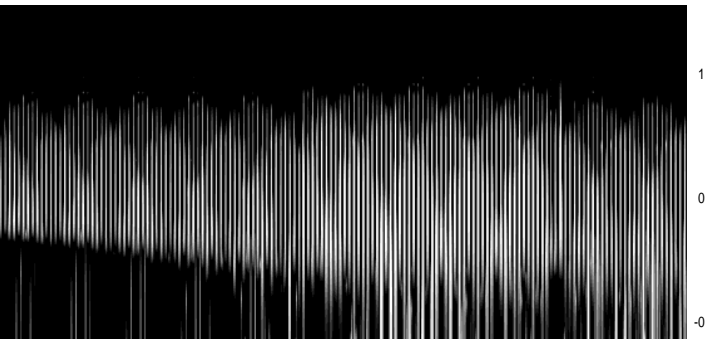

Figure 6(b)

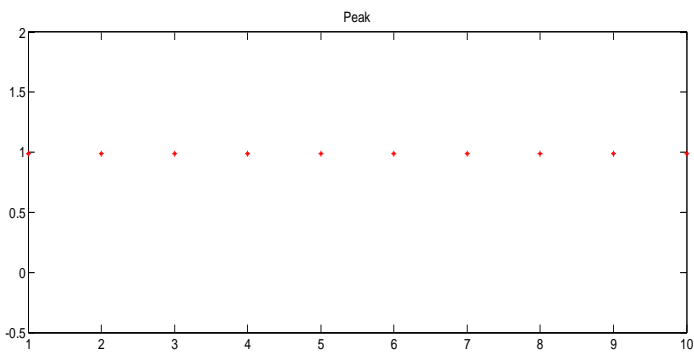

Figure 6(d)

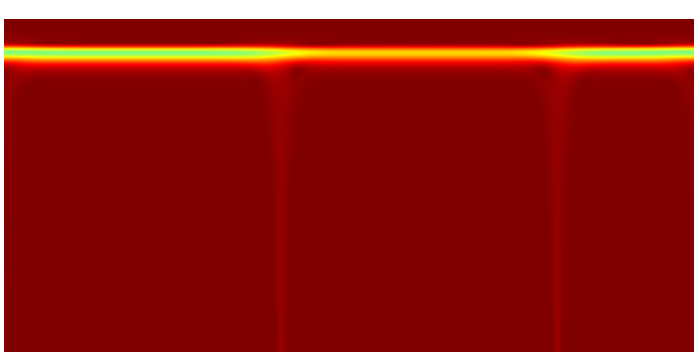

Figure 6(b)

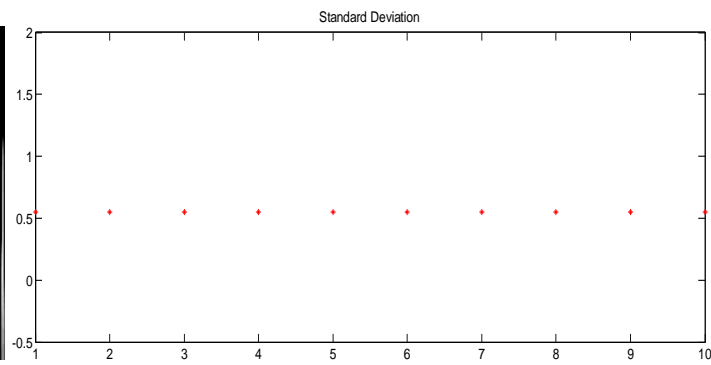

Figure 6(c)

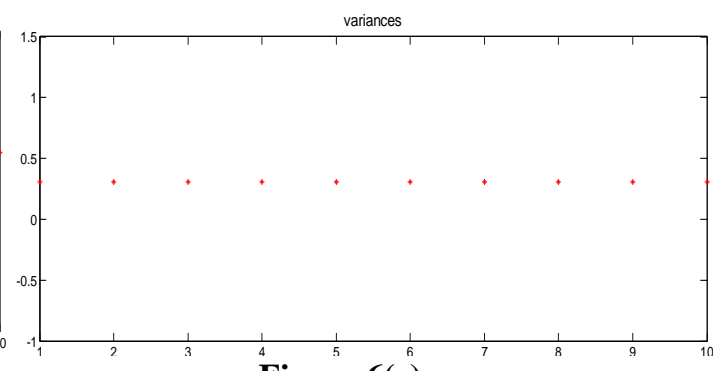

Figure 6(e)

Voltage swell causes the rise of $10-90 \%$ of the system voltage. It is generated by disconnecting the heavy load for 10 cycles. The duration of the swell disturbance is 0.2 to 0.4 cycles in $1 \mathrm{~min}$. The voltage swell waveform is shown in figure 7(a). The time frequency analysis of S-transform and the spectrogram representation are shown in the figures $7(\mathrm{~b})$ and $7(\mathrm{c})$. The standard deviation, peak value and the variances of the voltage swell are shown in the figures 7(d) and 7(f).

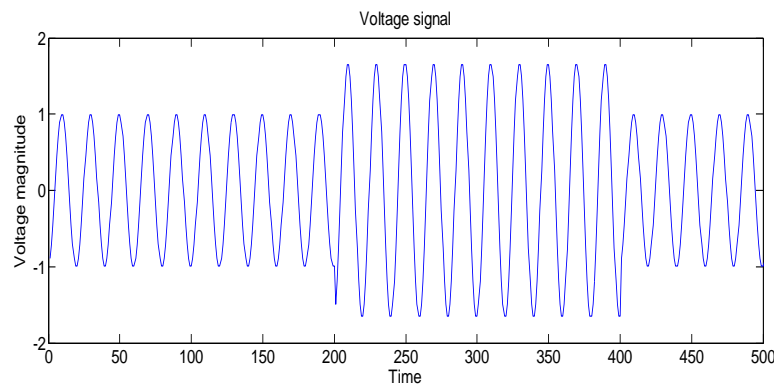

Figure 7(a)

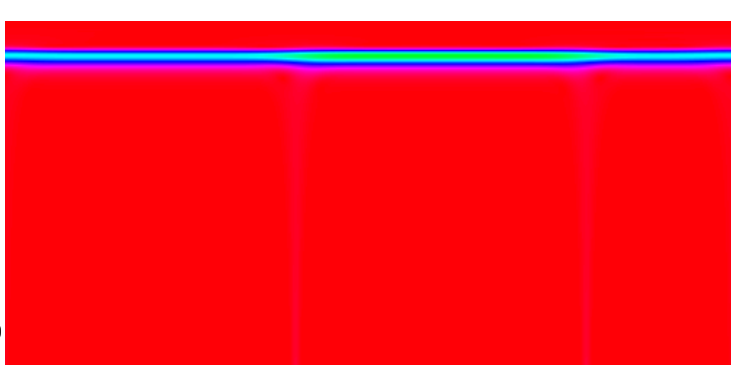

Figure 7(b) 


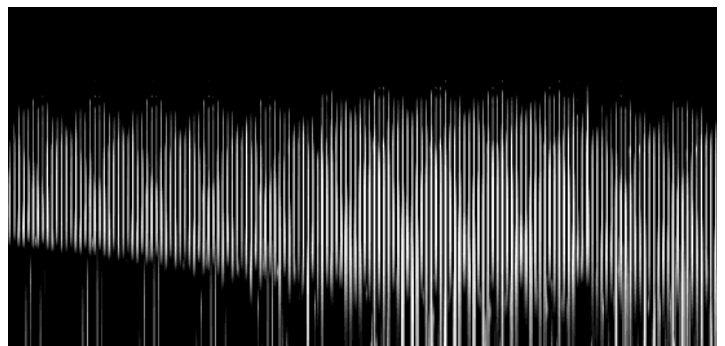

Figure 7(c)

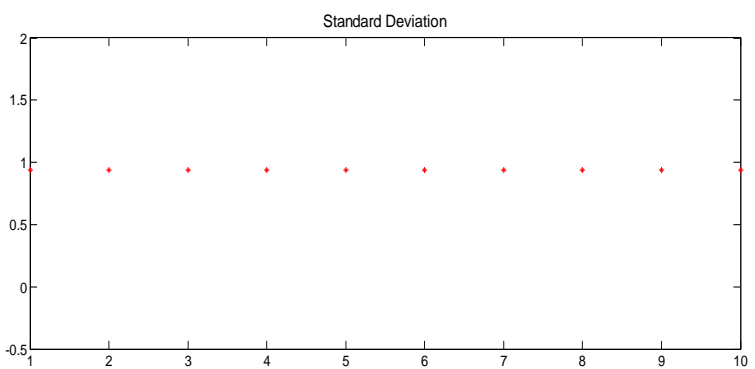

Figure 7(d)

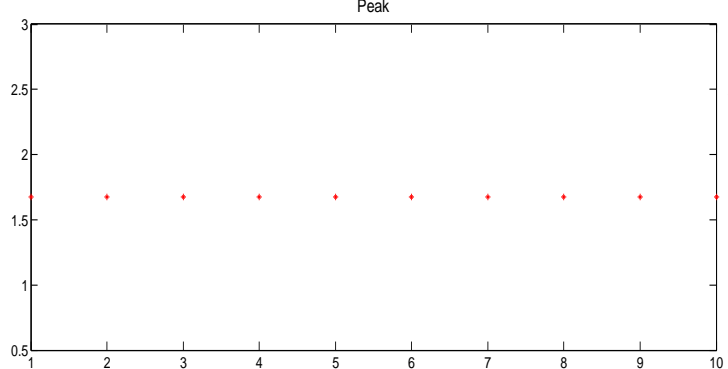

Figure 7(e)

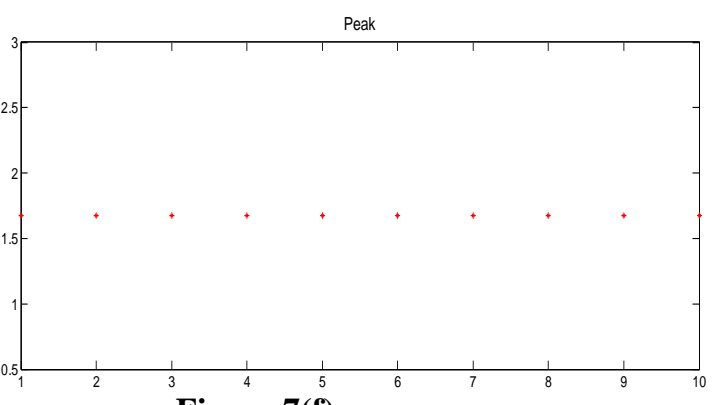

Figure 7(f)

Outages may be seen as a loss of voltage on the system for the duration of 0.5 cycles to $1 \mathrm{~min}$. An outage is generated by simulating a 3-phase dead short circuit to ground. The voltage waveform of an outage event is shown in the figure 8(a). The time frequency analysis of S-transform and the spectrogram representation are shown in the figures $8(\mathrm{~b})$ and $8(\mathrm{c})$. The standard deviation, peak value and the variances of the voltage swell are shown in the figures $8(\mathrm{~d})$ and $8(\mathrm{f})$.

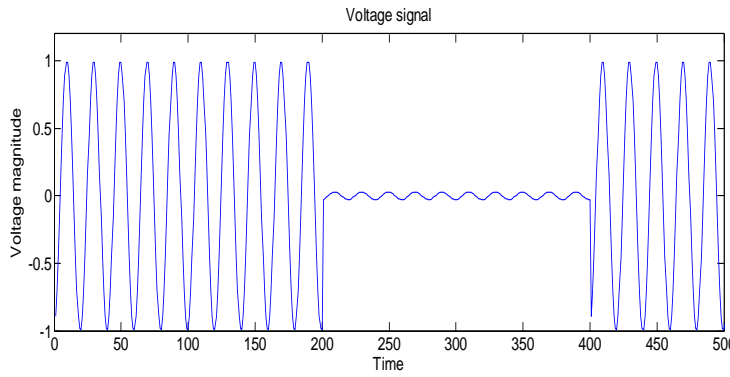

Figure 8(a)

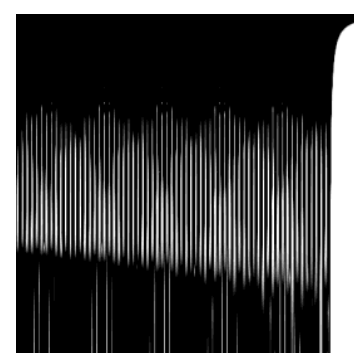

Figure 8(c)

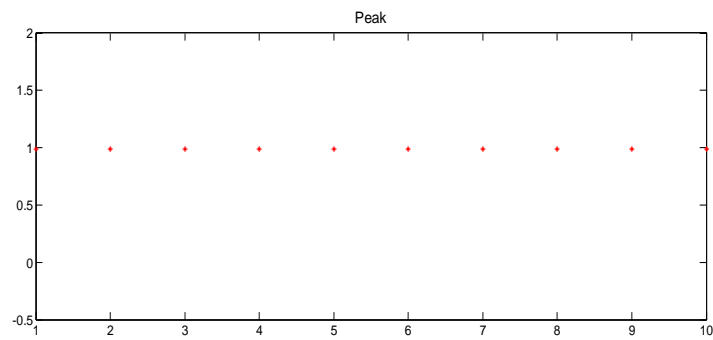

Figure 8(e)

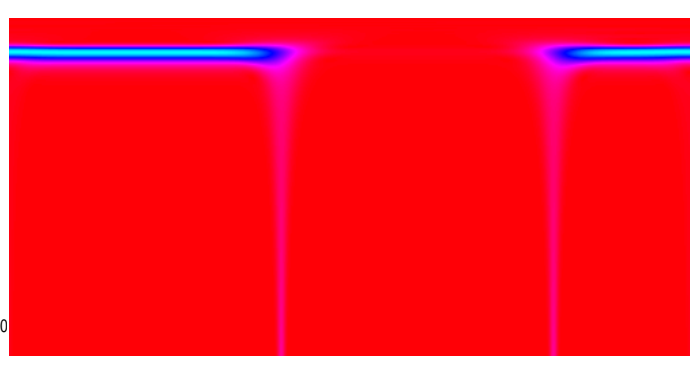

Figure 8(b)

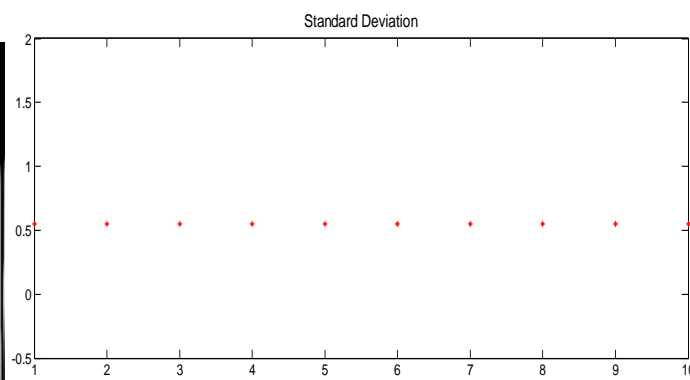

Figure 8(d)

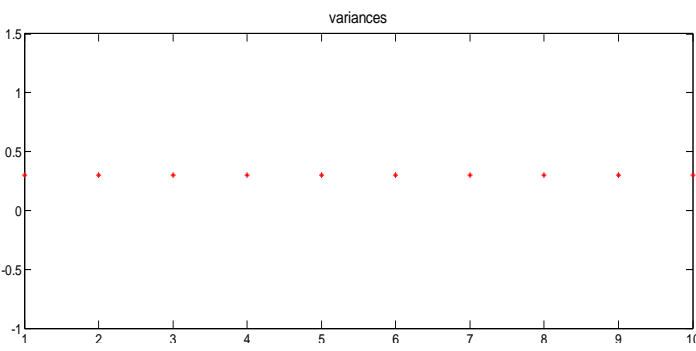

Figure 8(f) 
Harmonics are generated by connecting a non linear load to the system for 10 cycles. Figure 9(a) shows the distortion of voltage waveform and their corresponding time frequency analysis of S-transform and the spectrogram representation are shown in the figures 9(b) and 9(c). The standard deviation, peak value and variances of harmonics are shown in the figures $9(\mathrm{~d})$ and $9(\mathrm{f})$.

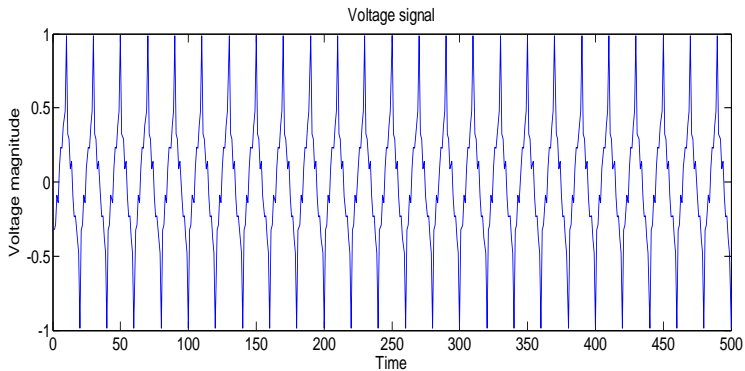

Figure 9(a)
Figure 9(b)

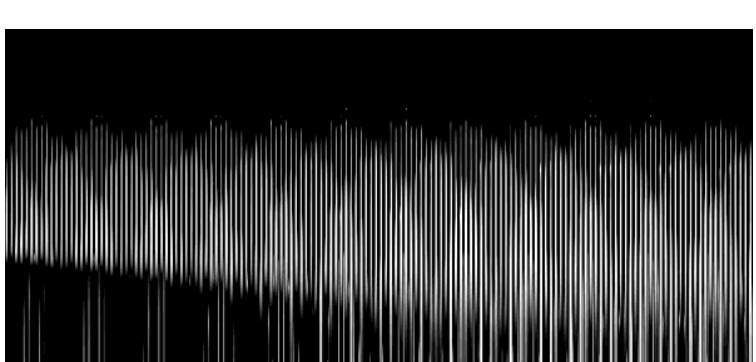

Figure 9(c)

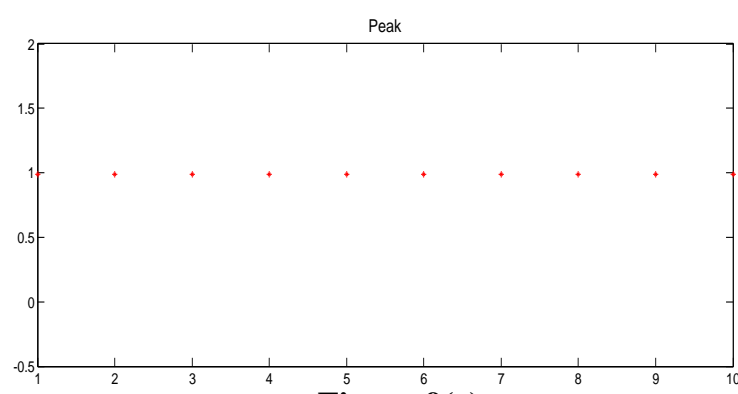

Figure 9(e)

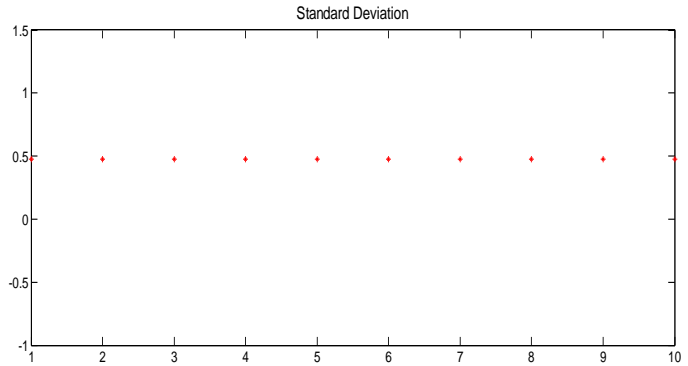

Figure 9(d)

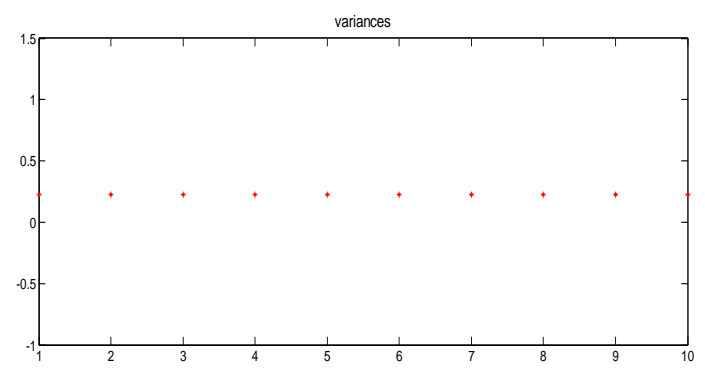

Figure 9(f)

Sag with harmonics are caused by the presence of a nonlinear load and occurrence of single line to ground fault for a duration of 0.2 to 0.4 cycles . The waveform which contains harmonic distortion with sag event is shown in the figure 11(a). The time frequency analysis of S-transform and the spectrogram representation of the harmonics are shown in the figures 11(b) and 11(c). The standard deviation, peak value and the variances of the harmonics are shown in the figures 11(d) and 11(f).

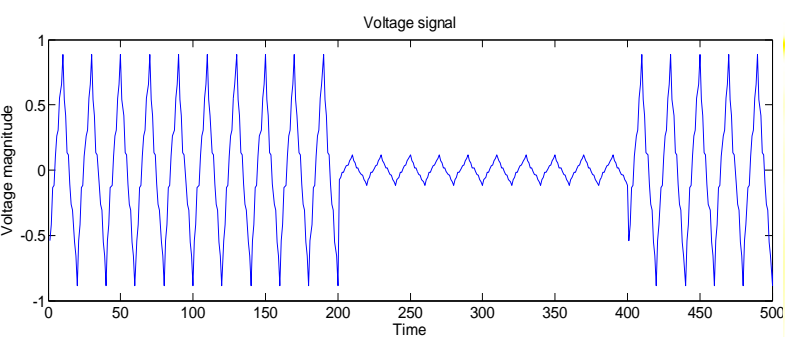

Figure 11(a)
Figure 11(b) 


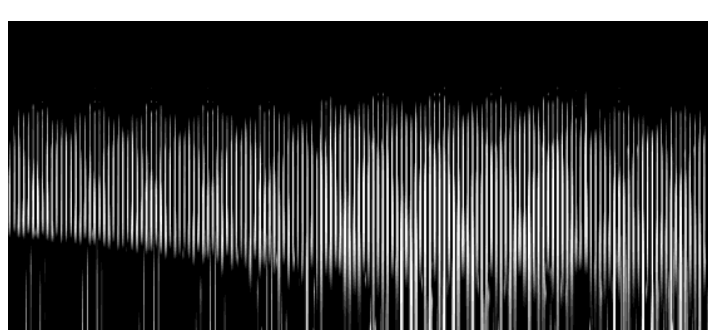

Figure 11(c)

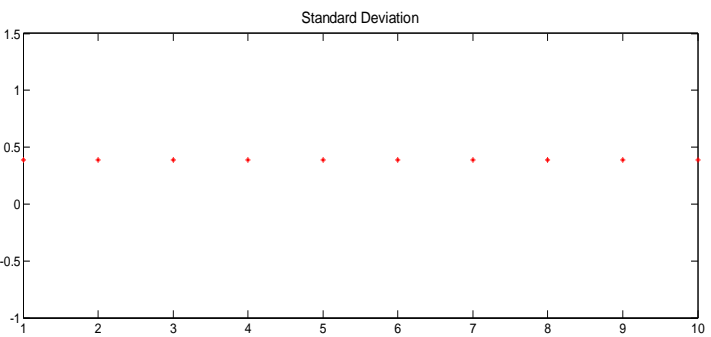

Figure 11(d)

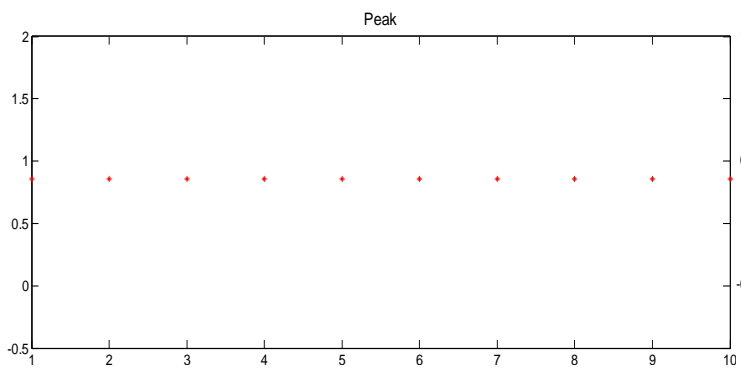

Figure 11(e)

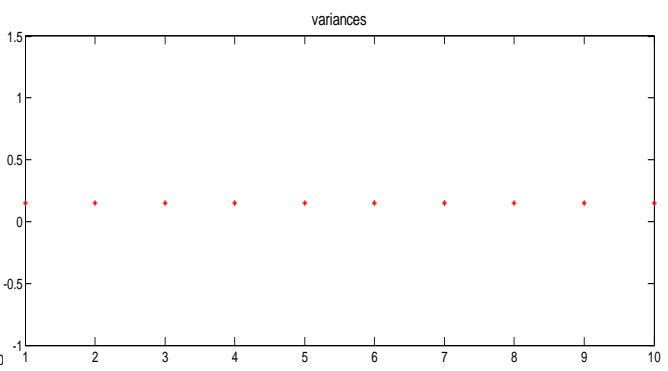

Figure 11(f)

Swell with harmonics is caused by the presence of nonlinear load and disconnecting the heavy load for 5 cycles in the duration of 0.2 to 0.4 cycles. The waveform for harmonic distortion with swell is shown in the figure 12(a). The time frequency analysis of S-transform and the spectrogram representation are shown in the figures $12(\mathrm{~b})$ and $12(\mathrm{c})$. The standard deviation, peak value and variances of the swell with harmonics are shown in the figures $12(\mathrm{~d}) \& 12(\mathrm{f})$.

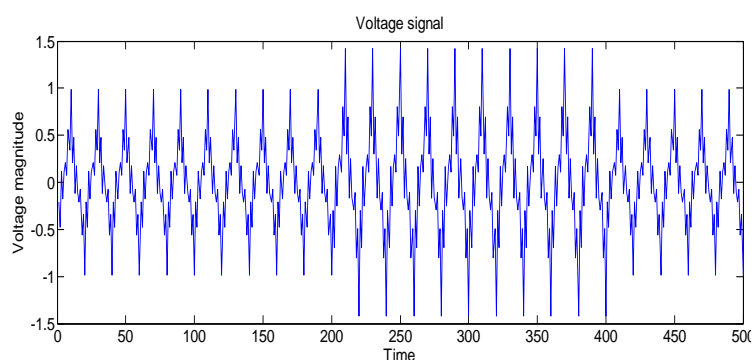

Figure 12(a)

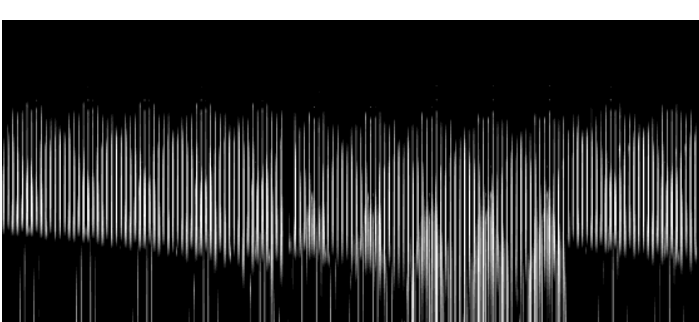

Figure 12(c)

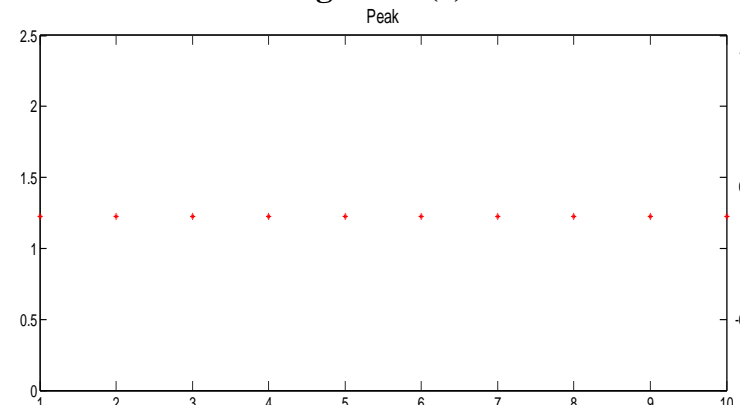

Figure 12(e)
Figure 12(b)

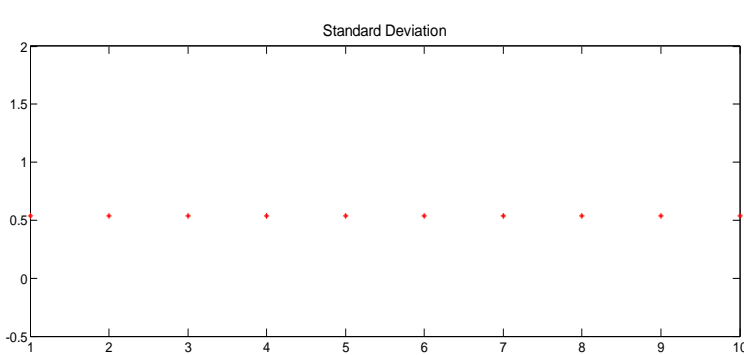

Figure 12(d)

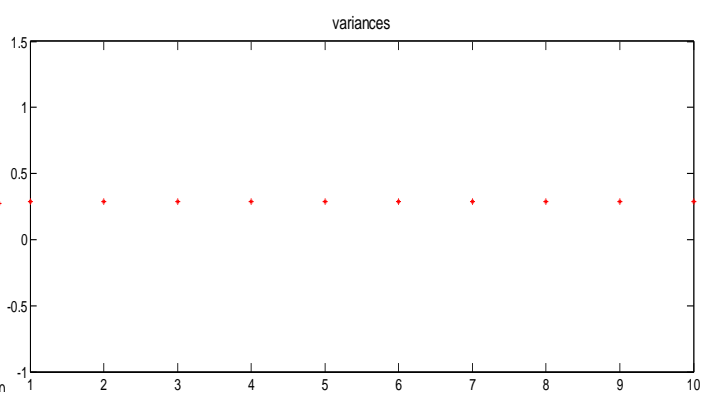

Figure 12(f) 
Flicker disturbance is caused by a continuous and rapid variation of the system load. It is simulated by the continuous connection and disconnection of the heavy load. The waveform of flicker is shown in the figure 13(a). The time frequency analysis of S-transform and the spectrogram representation of the flicker is shown in the figures 13(b) and 13(c). The standard deviation. Peak value and the variances of the flicker are shown in the figures $13(\mathrm{~d})$ and 13(f).

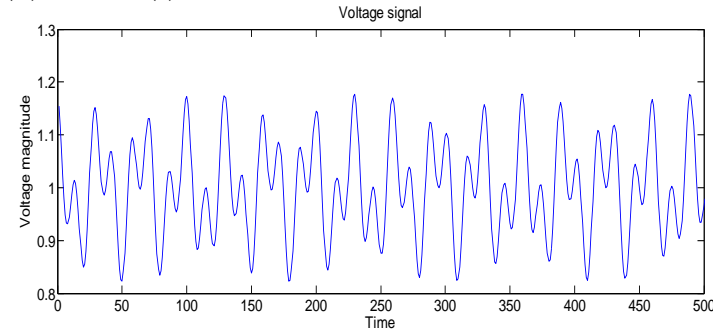

Figure 13(a)

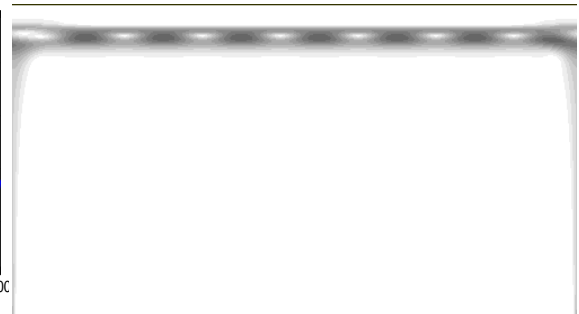

Figure 13(b)

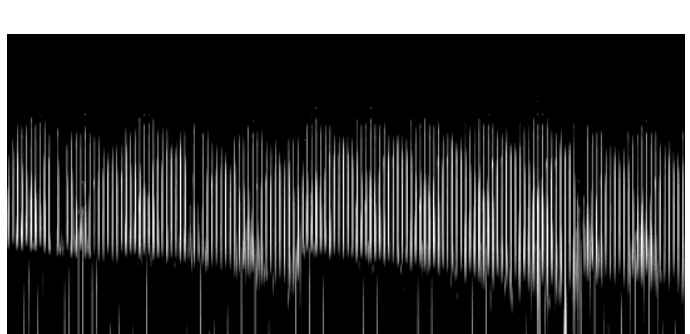

Figure 13(c)

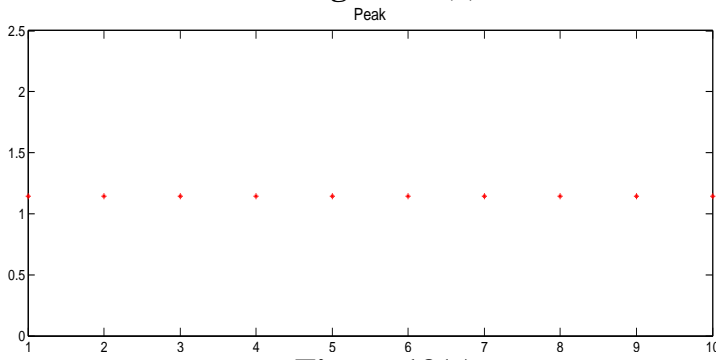

Figure 13(e)

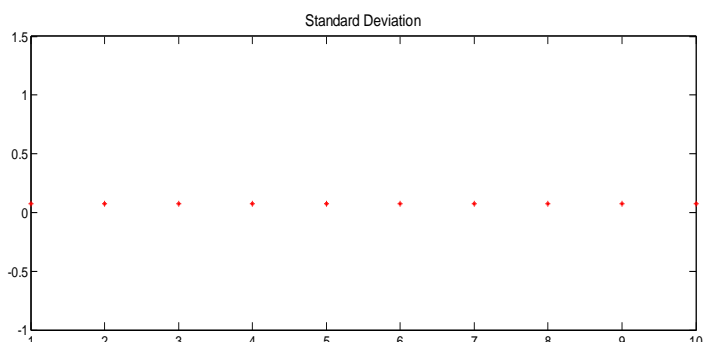

Figure 13(d)

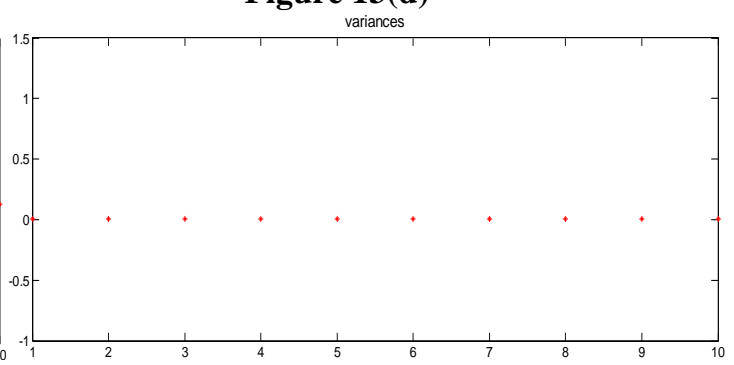

Figure 13(f)

Notch is a disturbance of the nominal power voltage waveform lasting for less than half a cycle. The disturbance is initially of opposite polarity and hence it is to be subtracted from the waveform. It is generated by the connection of the 3 phase non-linear load. The voltage notch waveform is shown in the figure 14(a). The time frequency analysis of S-transform and the spectrogram representation are shown in the figures 14(b) and 14(c). The standard deviation, peak value and variances of notch are shown in the figures 14(d) and 14(f).

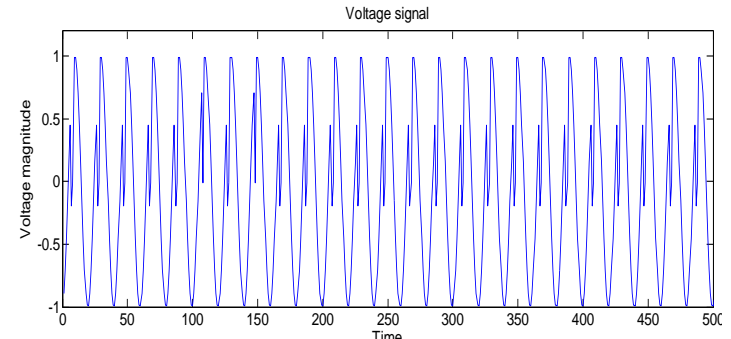

Figure 14(a)

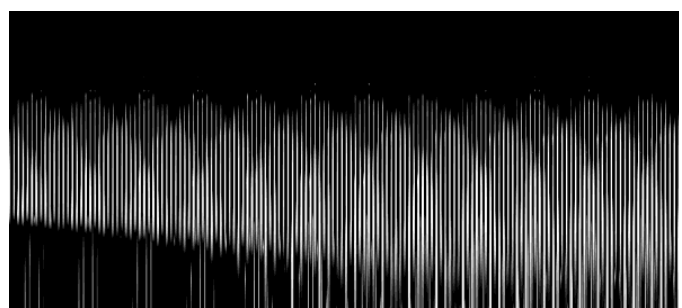

Figure 14(b)

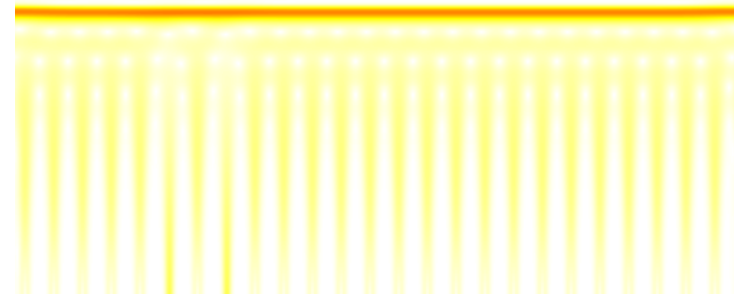

Figure 14(a)

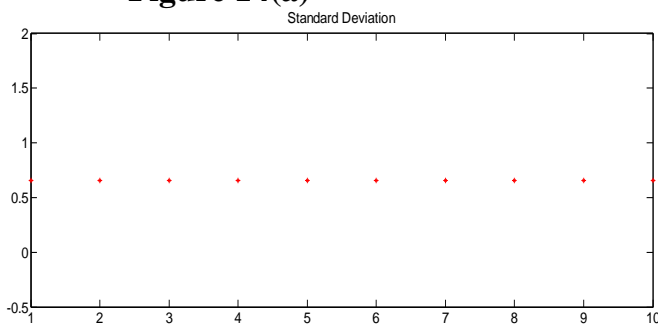

Figure 14(c) 


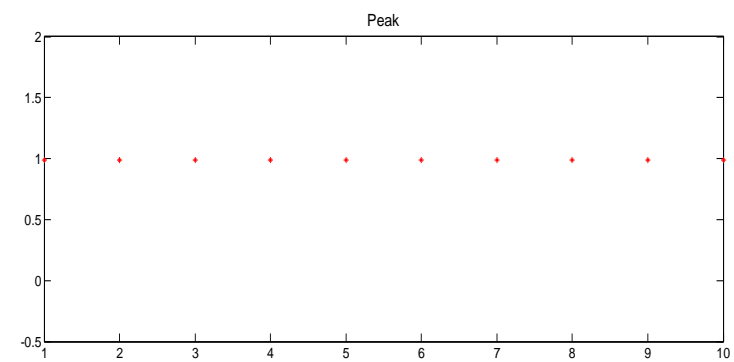

Figure 14(d)

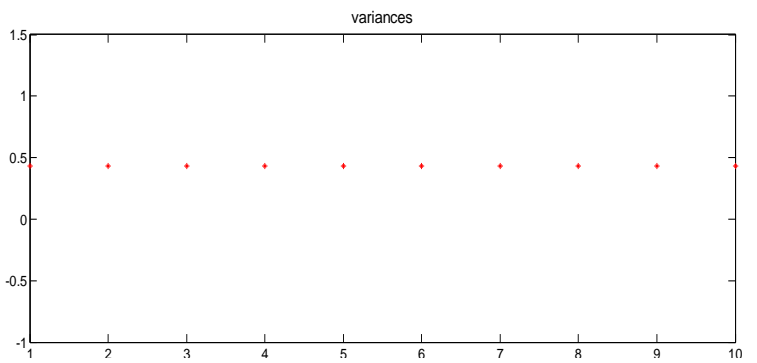

Figure 14(e)

Table 3.Classification accuracy

\begin{tabular}{|c|l|c|c|c|}
\hline Sno & Power Quality Disturbances & \multicolumn{3}{c|}{ Percentage of Accuracy } \\
\cline { 3 - 5 } & & Input Features & $\begin{array}{c}\text { S-transform based fuzzy expert } \\
\text { system }\end{array}$ & $\begin{array}{c}\text { S-transform based Neural } \\
\text { Network }\end{array}$ \\
\hline 1 & Pure Sine & 100 & 100 & 100 \\
\hline 2 & Voltage Sag & 100 & 98 & 100 \\
\hline 3 & Voltage Swell & 100 & 98 & 100 \\
\hline 4 & Outages & 100 & 98 & 98 \\
\hline 5 & Harmonics & 100 & 97 & 96 \\
\hline 6 & Sag with Harmonics & 100 & 98 & 96 \\
\hline 7 & Swell with Harmonics & 100 & 98 & 98 \\
\hline 8 & Flicker & 100 & 100 & 98 \\
\hline 9 & Notch & 100 & 100 & 98 \\
\hline Overall accuracy & & 98.56 & 98 \\
\hline
\end{tabular}

\section{Conclusion}

Detection and classification of the power quality disturbances has been done by the proposed technique S-transform and MLP based Neural Network. The Power quality disturbance waveforms were generated through Matlab simulation on the test system. Through S-transform method the input features such as standard deviation, variances and peak value were extracted and MLP based neural network has been applied for classifying the disturbances. The method enables the accurate classification of all nine types of power quality disturbances. Simulation results demonstrate the performance and accuracy of the S-transform technique. MLP based neural network has the wide range of skills that can be trained for any input combination and its application has been found to be particularly suitable for classification of disturbances of varying nature.

\section{References}

[1]. Surya Santoso, Edward J. Powers, and W. Mack Grady, "Power quality assessment via wavelet transform analysis", IEEE Transaction on power delivery, 1996.

[2]. P.K Dash,S.K Panda, A.C.Liew,B.Mishra,R.K.Jena, "A new approach to monitoring electric power quality", Electric power systems research, 1998.

[3]. Shyh-Jier Huang , Cheng-Tao Hsieh and Ching-Lien Huang, "Application of wavelets to classify power system disturbances", Electric power systems research,1998.

[4]. Mladen Kezunovic, "Advanced assessment of the power quality events", IEEE Transaction, 2000.

[5]. Surya Santoso, Edward J. Powers, W. Mack Grady, Jeff Lamoree and Siddharth C. Bhatt "Characterization of distribution power quality events with Fourier and wavelet transform analysis", IEEE Transaction on power delivery, 2000.

[6]. A.M.Gaouda, S.H.Kanoun, M.M.A.Salama, and A.Y.Chikhani, "Pattern recognition applications for power system disturbance classification", IEEE Transaction on power delivery, Vol 17, no.3, July 2002.

[7]. P.K Dash, G.Panda and B. K. Panigrahi, "Power quality analysis using S-transform", IEEE Transaction on power delivery, Vol 18, no.2, April 2003.

[8]. P.K Dash, I. W. C. Lee, "S-transform-based intelligent system for classification of power quality disturbance signals", IEEE Transaction on industrial electronics, Vol 50, no.4, August 2003

[9]. Inigo Monedero, Jorge Ropero, Antonio García, Jose Manuel Elena and Juan C. Montano, "Classification of electrical disturbances in real time using neural networks", IEEE transaction on power delivery, Vol 22, no.3, July 2007.

[10]. Abdul Rahim Abdullah, Ahmad Zuri Sha'ameri, Abd Rahim Mat Sidek and Mohammad Razman Shaari, "Detection and classification of power quality disturbances using time-frequency analysis technique”, IEEE transaction, 2007.

[11]. S. Mishra, C. N. Bhende, and B. K. Panigrahi, "Detection and classification of power quality disturbances using S-transform and Probabilistic neural network", IEEE transaction on power delivery, Vol 23, no.1, January 2008.

[12]. Sami Ekici, "Classification of power system disturbances using support vector machines", Expert systems with applications, 2009.

[13]. T.Jayasree, D.Devaraj, R.Sukanesh," Power quality disturbance classification using Hilbert transform and RBF networks", Neurocomputing , 2010

[14]. H.S. Behera, P.K. Dash, B. Biswal, "Power quality time series data mining using S-transform and fuzzy expert system", Applied soft computing, 2010.

[15]. Wenxin Liu, Il-YopChung, LiLiu, SiyuLeng, David A.Cartes, "Real-time particle swarm optimization based current harmonic cancellation", Engineering Applications of Artificial Intelligence, 2011.

[16]. Jinfeng Ren, and Mladen Kezunovic, "A hybrid method for power system frequency estimation", IEEE transaction on power delivery, Vol 27, no.3, July 2012. 
[17]. Faeza Hafiz, A. Hasib Chowdhury, and Celia Shahnaz, "An approach for classification of power quality disturbances based on Hilbert Huang transform and Relevance vector machine", IEEE transactions, 2012.

[18]. Okan Ozgonenela, Turgay Yalcina, Irfan Guneyb, and Unal Kurtc, "A new classification for power quality events in distribution systems", Electric power system research, 2013.

[19]. Yasemin onal, U.Cidem turnal, "The Orthogonal Hilbert-Huang transform application in voltage flicker analysis", IEEE transactions, 2013.

[20]. B. Biswal, M. Biswal, S. Mishra and R. Jalaja, "Automatic Classification of Power Quality Events Using Balanced Neural Tree", IEEE Transaction on Industrial electronics. Vol 61, January 2014. 